
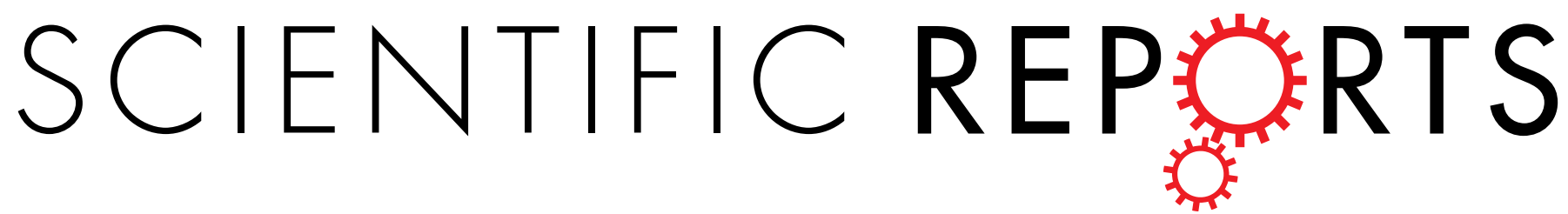

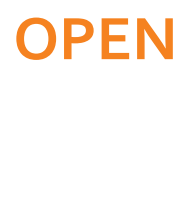

Received: 12 January 2016

Accepted: 19 January 2016

Published: 16 February 2016

\section{Altered drug susceptibility during host adaptation of a Plasmodium falciparum strain in a non-human primate model}

Nicanor Obaldía IIII, ${ }^{1,}$, Geoffrey S. Dow ${ }^{2}$, Lucia Gerena ${ }^{2}$, Dennis Kyle ${ }^{3}$, William Otero ${ }^{4}$, Pierre-Yves Mantel ${ }^{1}$, Nicholas Baro ${ }^{1}$, Rachel Daniels ${ }^{1}$, Angana Mukherjee ${ }^{1}$, Lauren M. Childs s,6, Caroline Buckee ${ }^{5,6}$, Manoj T. Duraisingh ${ }^{1}$, Sarah K. Volkman ${ }^{1,7,8}$, Dyann F. Wirth ${ }^{1,7}$ \& Matthias Marti ${ }^{1}$

Infections with Plasmodium falciparum, the most pathogenic of the Plasmodium species affecting man, have been reduced in part due to artemisinin-based combination therapies. However, artemisinin resistant parasites have recently emerged in South-East Asia. Novel intervention strategies are therefore urgently needed to maintain the current momentum for control and elimination of this disease. In the present study we characterize the phenotypic and genetic properties of the multi drug resistant (MDR) P. falciparum Thai C2A parasite strain in the non-human Aotus primate model, and across multiple passages. Aotus infections with C2A failed to clear upon oral artesunate and mefloquine treatment alone or in combination, and ex vivo drug assays demonstrated reduction in drug susceptibility profiles in later Aotus passages. Further analysis revealed mutations in the $p f c r t$ and $p f d h f r$ loci and increased parasite multiplication rate (PMR) across passages, despite elevated pfmdr 1 copy number. Altogether our experiments suggest alterations in parasite population structure and increased fitness during Aotus adaptation. We also present data of early treatment failures with an oral artemisinin combination therapy in a pre-artemisinin resistant $P$. falciparum Thai isolate in this animal model.

Antimalarial drug resistance is one of the greatest threats to the current malaria eradication agenda ${ }^{1}$. Oral artemisinin-based combination therapy (ACT) is the standard of care for uncomplicated malaria, while parenteral intravenous (i.v.) treatment is used in severe cases. Artemisinin (QHS) is the fast acting component of ACTs that accelerates clearing of young ring stage parasites by the spleen ${ }^{2}$. In 2009 decreased susceptibility to AS was first observed in western Cambodia ${ }^{3}$, and has since been detected in the rest of Southeast Asia, including recent reports of clinical treatment failure of ACTs such as artesunate/mefloquine (AS/MQ) or artemether/lumefantrine $(\mathrm{AL} \text { or Coartem })^{4,5}$. In vitro selection experiments followed by genome-wide association studies have meanwhile identified mutations in the PF3D7_1343700 (PF13_0238) genomic locus encoding a Kelch protein that are strongly correlated with slow clearing parasites ${ }^{6}$. Such mutations appear to occur in the context of a specific genetic background in Southeast Asia but so far have not been detected in Africa ${ }^{7,8}$.

The TM90C2A (C2A) parasite is a multidrug resistant $P$. falciparum strain originally isolated from a patient in Thailand in 1992, prior to the observation of altered susceptibility to QHS in Southeast Asia ${ }^{9}$. Initial genotyping

${ }^{1}$ Department of Immunology and Infectious Diseases, Harvard |T.H. Chan School of Public Health, Boston, MA, United States. ${ }^{2}$ Walter Reed Army Institute of Research, Silver Springs, MD, United States. ${ }^{3}$ Department of Global Health, University of South Florida, Tampa, FL, United States. ${ }^{4}$ Center for the Evaluation of Antimalarial Drugs and Vaccines, Tropical Medicine Research/Instituto Conmemorativo Gorgas de Estudios de la Salud, Panamá City, Republic of Panama. ${ }^{5}$ Center for Communicable Disease Dynamics and Harvard |T.H. Chan School of Public Health, Boston, MA, United States. 'Department of Epidemiology, Harvard |T.H. Chan School of Public Health, Boston, MA, United States. ${ }^{7}$ The Broad Institute of MIT and Harvard, Cambridge, MA, United States. ${ }^{8}$ School of Nursing and Health Sciences, Simmons College, Boston, MA United States. Correspondence and requests for materials should be addressed to N.O. (email: nobaldia@hsph.harvard.edu) or M.M. (email: mmarti@hsph.harvard.edu) 
demonstrated presence of quadruple-mutations in the dhfr-thymidylate synthase gene ${ }^{10}$ and increased $p f m d r 1$ copy number ${ }^{11}$. This combination of mutations and $p f m d r 1$ copy number variation $(\mathrm{CNV})$ has been associated in field isolates with higher in vitro inhibitory concentrations to MQ, quinine (QN), halofantrine and QHS, and with failure of MQ monotherapy and AS/MQ combination therapy at the Thai-Cambodian border ${ }^{12,13}$. Notably, AS/ MQ was deployed in 1994 in Thailand and adopted as standard treatment by the Thai health authorities in 2005. However, QHS monotherapy had been used in western Cambodia since the late 1970's and MQ was introduced in Thailand in $1984^{3}$.

Recently, we reported the adaptation of the Thai C2A clone to splenectomized Aotus l. lemurinus monkeys (Panamanian Owl monkey). Plasmodium infection in Aotus was first reported in Panama at the Gorgas Memorial Laboratory in 1966 and has since been established as a major non-human primate model to host-parasite-vector interactions, immunology and pathophysiology and evaluation of drugs and vaccines in $P$. falciparum and P. vivax ${ }^{14}$. During the adaptation process of passaging the parasite in Aotus, we observed decreasing susceptibility of the parasites to oral MQ or i.v. AS alone or in combination ${ }^{15}$. To determine the basis for the altered drug sensitivity phenotype during Aotus adaptation, we tested the antimalarial drug efficacy to MQ and artemisinin derivatives in vivo and in vitro in $\mathrm{C} 2 \mathrm{~A}$, and correlated these parameters with growth rates, genotypic changes and mutations in antimalarial drug resistance genes.

\section{Results}

Artesunate treatment failure with the C2A P. falciparum strain in the Aotus model. We previously demonstrated treatment failures upon AS treatment with the multidrug resistant C2A in the Aotus monkey model ${ }^{15}$. Specifically, we observed recrudescence in monkey passage VII and suppression in passage VIII, in each case after treatment with AS at $20 \mathrm{mg} / \mathrm{Kg}$ i.v. for three days ${ }^{15}$. To systematically investigate this drug susceptibility phenotype we performed a controlled experiment in Aotus and followed parasitemia upon oral AS treatment alone, or in combination with MQ. Malaria naive and splenectomized male and female Aotus lemurinus lemurinus monkeys were inoculated with $5 \times 10^{6}$ parasites of the C2A clone on its IX serial passage from a donor Aotus and further divided into three oral treatment groups of two monkeys each (Fig. 1, Table 1): one group was treated with a single dose of $40 \mathrm{mg} / \mathrm{Kg}$ of oral MQ; a second group was treated with daily doses of $33 \mathrm{mg} / \mathrm{Kg}$ of oral AS for three days; and a third group was treated with a combination of a single dose of oral MQ and three daily doses of oral AS. As a control a spleen intact animal was also infected and treated with the same dose regimen as the third group. Parasitemia was patent in all animals between days 1-2 post infection (PI), reaching peak parasitemia of $>100,000$ parasites/ $\mu \mathrm{L}$ in groups $1-3$ by day $9-11$ PI. In contrast, a peak parasitemia of 340 parasites $/ \mu \mathrm{L}$ on day $16 \mathrm{PI}$ was reached in the spleen intact animal. On the first day $>100,000$ parasites $/ \mu \mathrm{L}$ were reached treatment was initiated (Day 0 in Fig. 1).

In each of the three treatment regimes, the animals remained parasitemic with $10-100$ parasites $/ \mu \mathrm{L}$ for $>10$ days (Fig. 1A, Table 1 and S3). In the spleen intact animal, parasitemia persisted at low levels until treatment on day 16 PI and was cleared by day 3 of treatment (Fig. 1B). In contrast, when splenectomized animals were infected with parasites from Aotus passages III and IV and treated i.v. with AS at $33 \mathrm{mg} / \mathrm{Kg}$ for 3 days and MQ at $40 \mathrm{mg} / \mathrm{Kg}$ once at peak parasitemia $(<100,000$ parasites $/ \mu \mathrm{L})$, parasites were cleared on average on day 5 following initiation of treatment (Fig. 1C, Table S3). Similar clearance times are found in human cohorts: in one study $25 \%$ of patients with parasitemia $>4 \%(>100,000$ parasites $/ \mu \mathrm{L})$ were positive beyond day 3 of treatment, but only $5 \%$ with parasitemia $<4 \%{ }^{16}$. Notably, the i.v. AS dosage used in our study is about eight-fold higher than what is commonly given to humans for oral treatment of uncomplicated ${ }^{17,18}$ or severe malaria $(33 \mathrm{mg} / \mathrm{Kg} \text { versus } 4 \mathrm{mg} / \mathrm{kg})^{19}$. Converting the Aotus animal dose of AS used in this study $(33 \mathrm{mg} / \mathrm{Kg})$ to a human equivalent dose based on body surface are ${ }^{20}$ would result in $\sim 11 \mathrm{mg} / \mathrm{Kg}$, approximately three times the human standard dose of $4 \mathrm{mg} / \mathrm{Kg}$. Together these experiments demonstrate that infections with the MDR C2A isolate in the Aotus/P. falciparum non-human primate model show characteristics of MQ resistance and reduced AS susceptibility, including parasite suppression upon i.v. or oral AS at $33 \mathrm{mg} / \mathrm{Kg} \times$ three days, or in combination with MQ at $40 \mathrm{mg} / \mathrm{Kg}$ once (Fig. 1).

C2A shows altered drug susceptibility profiles ex vivo. To further investigate the drug resistance profile of the $\mathrm{C} 2 \mathrm{~A}$ strain we performed ex vivo assays of $\mathrm{C} 2 \mathrm{~A}$ from several Aotus passages with a series of antimalarial compounds. Specifically, we determined the ex vivo $\mathrm{IC}_{50}$ values for Aotus passages II and X and the original in vitro-adapted TM90C2A isolate against MQ, CQ, atovaquone (ATV), QHA, DHA and AS (Fig. 2). As a control we also measured the $\mathrm{IC}_{50}$ of the sensitive D6 strain (CQ and MQ susceptible). These experiments confirmed that TM90C2A is indeed resistant to MQ, CQ and ATV (Fig. 2A), as observed in our previous experiments in the Aotus model ${ }^{15}$. MQ IC I0s $_{\text {s }}$ are $>30 \mathrm{nM}$, a concentration that has been associated with treatment failures in infected patients ${ }^{21,22}$. Interestingly we also observed reduced susceptibility with $\mathrm{C} 2 \mathrm{~A}$ to the three different artemisinin derivatives, QHA, DHA and AS, and compared to D6 (Fig. 2B). IC $_{50}$ concentrations were above the previously defined thresholds of $12 \mathrm{nM}^{21}$ for DHA (C2A passage X), and $20 \mathrm{nM}^{21,23}$ for AS (C2A passages II and $\mathrm{X}$ ). Interestingly, parasites showed a significant decrease in susceptibility against MQ, QHS and DHA between in vitro-adapted TM90C2A and C2A passages II and X. In contrast, CQ IC ${ }_{50}$ was not significantly different across $\mathrm{C} 2 \mathrm{~A}$ passages. To further evaluate possible reduction in artemisinin susceptibility across $\mathrm{C} 2 \mathrm{~A}$ passages, we performed the recently established ring stage assay (RSA) ${ }^{24}$. This experiment indeed demonstrated some reduced susceptibility for ex vivo C2A passages II and X compared to the in vitro-adapted original TM90C2A isolate and the D6 control (Fig. 2C), although still below the defined threshold of $1 \%$ ring stage survival for resistance ${ }^{24}$. Altogether these data demonstrated phenotypic changes in C2A across Aotus passages such as altered susceptibility to QHS, DHA, and MQ. Interestingly the in vitro-adapted TM90C2A did not show any signs of altered artemisinin susceptibility while being equally resistant to MQ and CQ compared to the Aotus passages. 

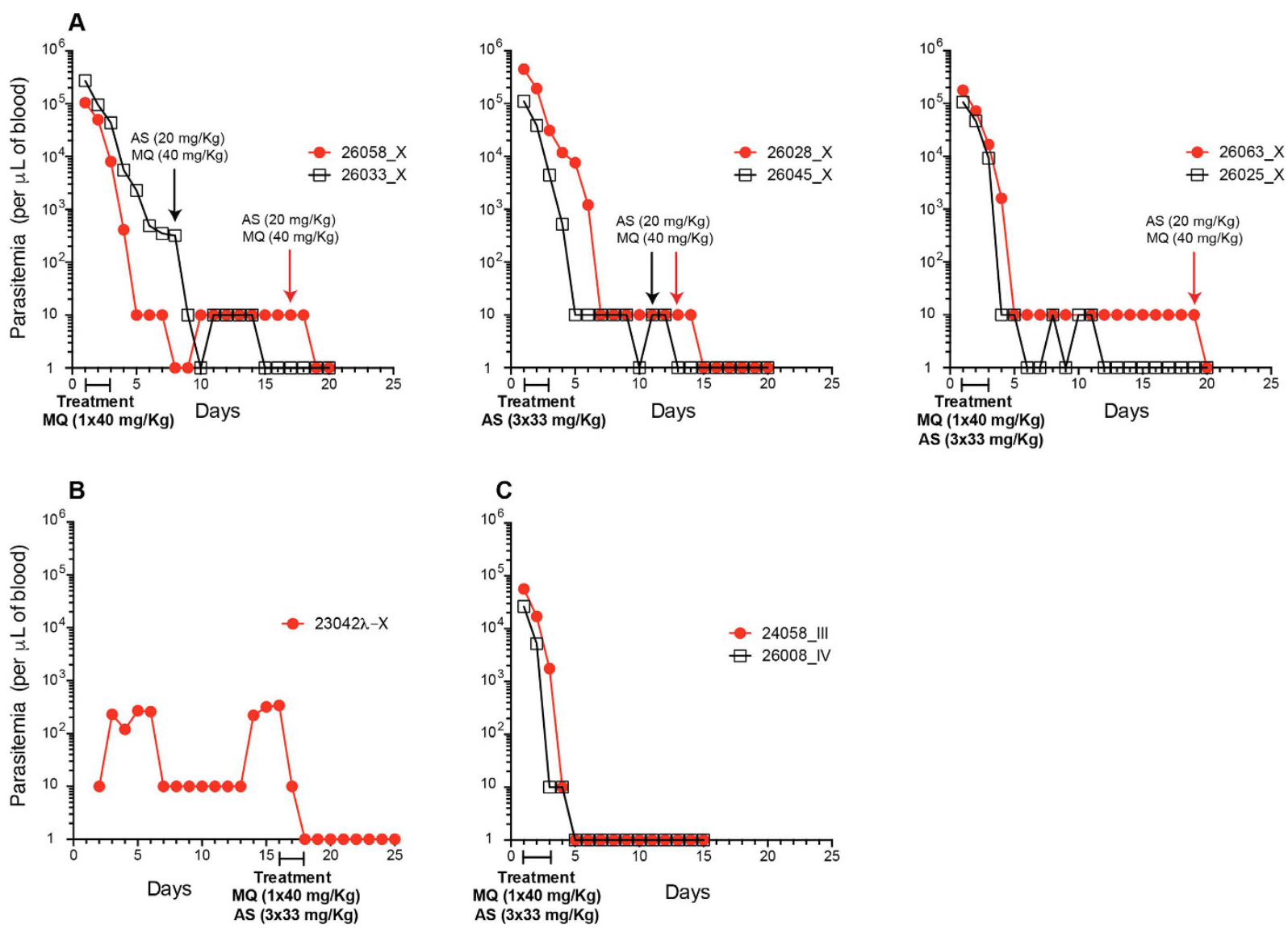

Figure 1. Antimalarial drug responses of Plasmodium falciparum C2A infected Aotus monkeys.

(A) Parasitemia response plots of Aotus monkeys infected with the P. falciparum C2A clone passage X. Animals were inoculated with $5 \times 10^{6}$ infected red blood cells from a donor monkey (MN26006, passage IX) and treated when parasitemia reached $>10^{5}$ parasites $\times \mu \mathrm{L}$ of blood. Panel (A) shows group 1 animals treated with MQ at $40 \mathrm{mg} / \mathrm{Kg}$ orally once, group 2 animals treated with AS at $33 \mathrm{mg} / \mathrm{Kg}$ orally $\times$ three days and group 3 animals treated with MQ and AS at $33 \mathrm{mg} / \mathrm{Kg} \times$ three days and MQ at $40 \mathrm{mg} / \mathrm{Kg}$ orally once. Panel (B) shows a spleen intact control treated with AS at $33 \mathrm{mg} / \mathrm{Kg} \times$ three days $+\mathrm{MQ}$ at $40 \mathrm{mg} / \mathrm{Kg}$ orally once on day 16 of infection. Panel (C) shows parasitemia responses of MN24058 and MN26008 inoculated with PfC2A passages III and IV respectively and treated with $\mathrm{AS}$ at $33 \mathrm{mg} / \mathrm{Kg} \times$ three days i.v. $+\mathrm{MQ}$ at $40 \mathrm{mg} / \mathrm{Kg}$ orally once.

\begin{tabular}{|c|c|c|c|c|c|c|c|c|c|c|c|c|}
\hline \multirow[b]{2}{*}{ Group } & \multirow[b]{2}{*}{ ID } & \multirow{2}{*}{$\begin{array}{c}\text { Aotus } \\
\text { Sex }\end{array}$} & \multirow[b]{2}{*}{$\begin{array}{c}\text { Weight } \\
\text { g. }\end{array}$} & \multirow{2}{*}{\begin{tabular}{|c} 
Parasitemia \\
Peak \\
$103 \times \mu \mathrm{L}$
\end{tabular}} & \multirow{2}{*}{$\begin{array}{c}\text { Day } \\
\text { of } \\
\text { peak }\end{array}$} & \multirow[b]{2}{*}{ Group } & \multirow[b]{2}{*}{$\begin{array}{c}\text { Regimen } \\
\mathrm{mg} / \mathrm{Kg}\end{array}$} & \multirow[b]{2}{*}{ Route } & \multirow{2}{*}{$\begin{array}{c}\text { Treatment } \\
\begin{array}{c}\text { Duration } \\
\text { days }\end{array}\end{array}$} & \multirow[b]{2}{*}{$\begin{array}{c}\text { Day of } \\
\text { treatment }\end{array}$} & \multirow[b]{2}{*}{$\begin{array}{c}\text { Day } \\
\text { rescue }\end{array}$} & \multirow[b]{2}{*}{$\begin{array}{c}\text { Results } \\
\text { primary }\end{array}$} \\
\hline & & & & & & & & & & & & \\
\hline 1 & 26058 & 2 & 829 & 104.60 & 11 & MQ & 40 & p.o. & 1 & 11 & 14 & Failed \\
\hline 1 & 26033 & 1 & 758 & 272.74 & 9 & MQ & 40 & p.o. & 1 & 9 & 5 & Failed \\
\hline 2 & 22028 & 2 & 764 & 449.73 & 9 & AS & 33 & p.o. & 3 & 9 & 10 & Failed \\
\hline 2 & 26045 & 1 & 789 & 110.58 & 11 & AS & 33 & p.o. & 3 & 11 & 8 & Failed \\
\hline 3 & 26063 & 1 & 782 & 177.84 & 9 & $\mathrm{AS}+\mathrm{MQ}$ & $33+40$ & p.o. & $3+1$ & 9 & 16 & Failed \\
\hline 3 & 26025 & 2 & 785 & 106.02 & 10 & $\mathrm{AS}+\mathrm{MQ}$ & $33+40$ & p.o. & $3+1$ & 10 & None & Failed \\
\hline 4 & $23042 \lambda$ & 1 & 724 & 0.340 & 16 & $\mathrm{AS}+\mathrm{MQ}$ & $33+40$ & p.o. & $3+1$ & 16 & None & Cured \\
\hline
\end{tabular}

Table 1. Parasitological treatment responses of Aotus monkeys infected with a Plasmodium falciparum C2A clone. Male $=1$; Female $=2 . \mathrm{MQ}=$ mefloquine; $\mathrm{AS}=$ artesunate. $\lambda=$ spleen intact. p.o. $=$ per os.

Genetic analysis of C2A during Aotus passages and in vitro adaptation. To track possible changes in the genetic composition of $\mathrm{C} 2 \mathrm{~A}$ across Aotus passages and during in vitro adaptation we first analyzed copy number variation (CNV) of $p f m d r 1$ (Fig. 2D). These experiments demonstrated variation in $p f m d r 1$ copy number between 2 and 2.5 across all passages, and copy number of 2 in the in vitro-adapted line, suggesting presence of mixed genotypes across Aotus passages.

Given the pfmdrl CNV data and the observed drug resistant profiles with C2A, we performed detailed genetic analysis of $\mathrm{C} 2 \mathrm{~A}$ across passages and with the in vitro-adapted TM90C2A. To determine whether $\mathrm{C} 2 \mathrm{~A}$ represented 
A
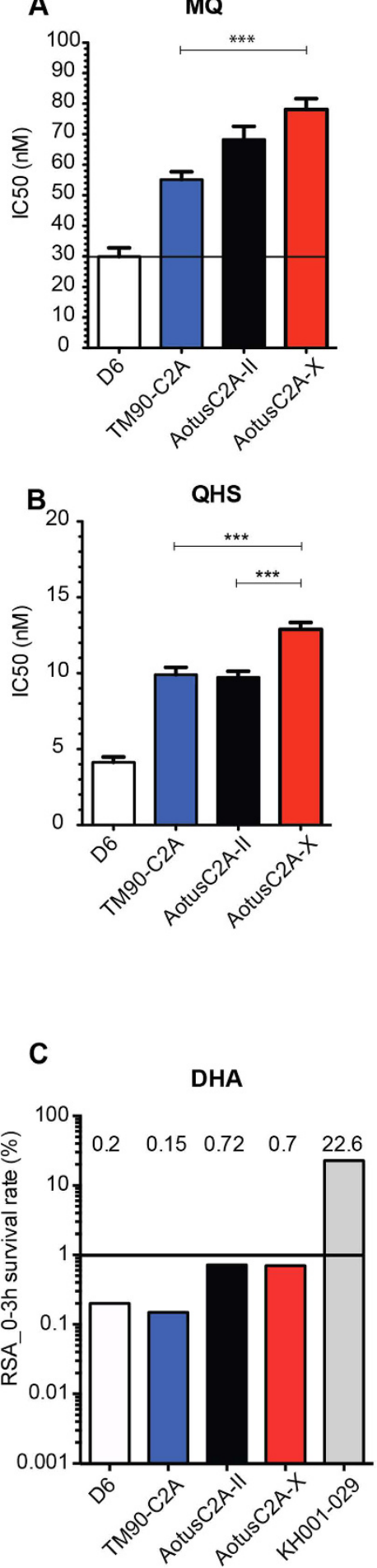

CQ

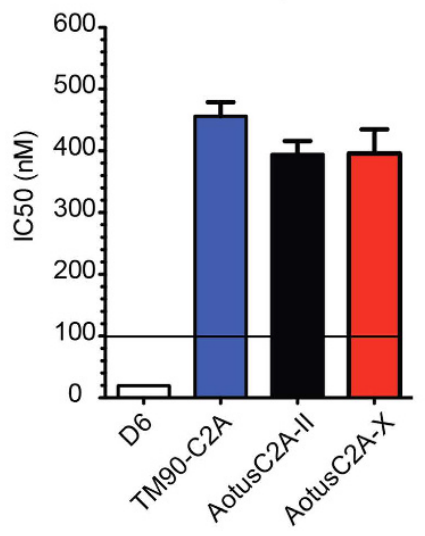

DHA

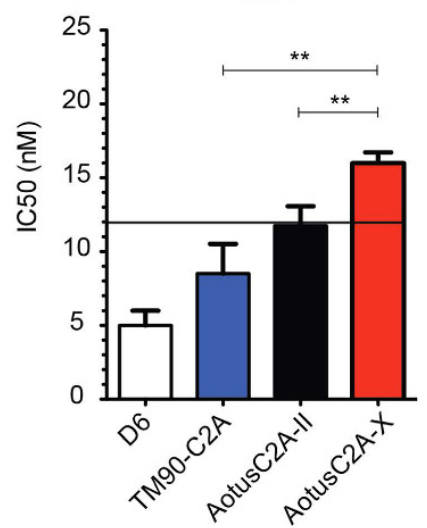

ATV

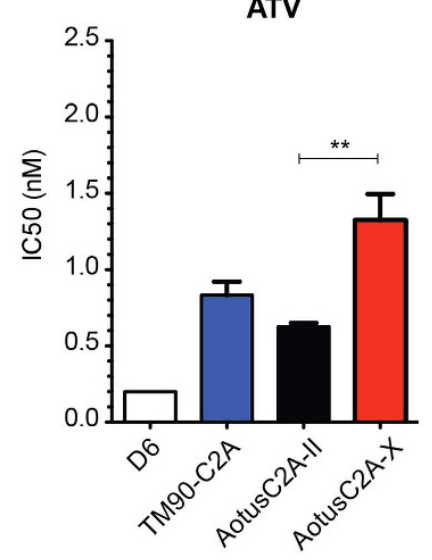

AS

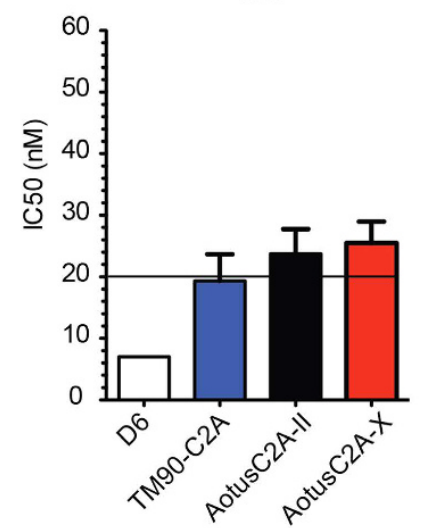

Figure 2. Ex vivo/in vitro antimalarial drug susceptibility and Pfmdr1 copy number variation of PfC2A across Aotus passages. $(\mathbf{A}, \mathbf{B}) \mathrm{IC}_{50}$ antimalarial drug $\mathrm{nM}$ concentration bar plots of reference strains D6 (white bar), in vitro culture adapted TM90C2A (blue bar) and $p f C 2 \mathrm{~A}$ Aotus adapted passage levels II (black bar) and $\mathrm{X}$ (red bar). Drug IC50 nM resistance threshold (black line). Mean \pm sample SEM (see also Table S1). (C) DHA drug susceptibility based on RSA. Survival percentage $=$ DHA treated parasites/DMSO treated parasites $\times$ 100. Red bar represents survival of artemisinin resistant positive control strain KH001-029, D6 is included as negative reference strain. Significant survival threshold (black line) is set at $1 \%$ as in Witkowski et al. ${ }^{24}$.

(D) Passage level pfmdr1 copy number fold change of a Plasmodium falciparum C2A clone during adaption to Aotus monkeys. P. falciparum strains with two $p f m d r 1$ gene copies are considered resistant to MQ.

Mean \pm sample SEM. Dashed line indicates threshold for $p f m d r 1 \mathrm{CN}$ level indicative of MQ resistance. Dotted line indicates threshold for $p f m d r 1 \mathrm{CN}$ level indicative of MQ sensitivity. $\mathrm{MQ}=$ Mefloquine; $\mathrm{CQ}=\mathrm{Chloroquine}$; $\mathrm{ATV}=$ Atovaquone; $\mathrm{AS}=$ Artesunate; $\mathrm{QHS}=$ Artemisinin; DHA = Dihydroartemisinin. $p=$ Mann-Whitney U significance $t$ test unpaired samples. ${ }^{\star * *} p<0.005 ;{ }^{* *} p<0.05$. 


\section{A Barcode assays}

\begin{tabular}{|c|c|c|c|c|c|c|c|c|c|c|c|c|c|c|c|c|c|c|c|c|c|c|c|c|c|c|}
\hline ID & Passage level & Passage history & 1 & 2 & 3 & 4 & 5 & 6 & 7 & 8 & 9 & 10 & 11 & 12 & 13 & 14 & 15 & 16 & 17 & 18 & 19 & 20 & 21 & 22 & 23 & 24 \\
\hline TM90C2A & nd & WRAIR-Culture & & $\mathrm{A}$ & $\mathrm{C}$ & $\bar{T}$ & $\mathrm{C}$ & $\bar{G}$ & $\mathrm{G}$ & $\mathrm{G}$ & $\mathrm{A}$ & & \begin{tabular}{|l|l}
$\mathrm{C}$ & \\
\end{tabular} & $\mathrm{G}$ & & $\mathrm{A} \mid$ & \begin{tabular}{|l|l}
$\mathrm{A}$ \\
\end{tabular} & \begin{tabular}{|l|l}
$\mathrm{C}$ \\
\end{tabular} & \begin{tabular}{c|c} 
\\
\end{tabular} & A & $\mathrm{C}$ & A & C & $T$ & $\mathrm{G}$ & $T$ \\
\hline MN92015 & II & 1th passage culture & & A & C & $T$ & C & $\bar{G}$ & $\bar{G}$ & $\mathrm{G}$ & $\mathrm{A}$ & & \begin{tabular}{|l|l}
$\mathrm{C}$ \\
\end{tabular} & $\mathrm{G}$ & & A & A & \begin{tabular}{|l|l} 
C \\
\end{tabular} & C & A & $\mathrm{C}$ & $\mathrm{A}$ & C & & G & $\bar{T}$ \\
\hline MN24058 & IIII & Aotus original & & $\mathrm{A}$ & C & T & $\mathrm{C}$ & $\bar{G}$ & $\mathrm{~N}$ & $\mathrm{G}$ & $\mathrm{A}$ & & \begin{tabular}{|l|l}
$\mathrm{C}$ \\
\end{tabular} & $G$ & & $\mathrm{~A}$ & A & \begin{tabular}{|l|l}
$\mathrm{C}$ \\
\end{tabular} & $c \mid$ & $\mathrm{A}$ & $\mathrm{C}$ & $\mathrm{A}$ & C & & $G$ & $T$ \\
\hline MN24058 & III & 5th passage culture & & A & C & 1 & $\mathrm{C}$ & $\bar{G}$ & $\mathrm{~N}$ & $\mathrm{G}$ & $\mathrm{A}$ & & \begin{tabular}{|l|l} 
C \\
\end{tabular} & $\mathrm{G}$ & & A & A & \begin{tabular}{|l|l} 
C \\
\end{tabular} & C & A & $\mathrm{C}$ & A & C & & G & $T$ \\
\hline MN93014 & IV & 1th passage culture & & $\mathrm{A}$ & C & $T$ & $\mathrm{C}$ & $\mathrm{G}$ & $\mathrm{N}$ & $\mathrm{G}$ & $A$ & & \begin{tabular}{|l|l}
$\mathrm{C}$ \\
\end{tabular} & $\mathrm{G}$ & 7 & A & A & \begin{tabular}{|l|l}
$\mathrm{C}$ \\
\end{tabular} & C & $\mathrm{A}$ & $\mathrm{C}$ & $\mathrm{A}$ & C & $\mathrm{T}$ & $\mathrm{G}$ & $\mathrm{N}$ \\
\hline MN12801 & $\mathrm{V}$ & Aotus original & & $\mathrm{A}$ & C & $T$ & - & $\bar{G}$ & $\mathrm{G}$ & $\mathrm{G}$ & $\mathrm{A}$ & & \begin{tabular}{|l|}
$\mathrm{C}$ \\
\end{tabular} & $\mathrm{G}$ & & $\mathrm{A}$ & A & \begin{tabular}{|l|l}
$\mathrm{C}$ \\
\end{tabular} & c & A & $\mathrm{C}$ & A & $c$ & $\bar{T}$ & $G$ & $\mathrm{~N}$ \\
\hline MN12907 & $\mathrm{VI}$ & Aotus original & & A & C & T & $\mathrm{C}$ & $\mathrm{G}$ & $\mathrm{G}$ & $\mathrm{G}$ & A & & \begin{tabular}{|l|l}
$c$ \\
\end{tabular} & $\mathrm{G}$ & & $\mathrm{A}$ & \begin{tabular}{|l|} 
A \\
\end{tabular} & \begin{tabular}{|l|l|}
$c$ \\
\end{tabular} & c & A & $\mathrm{C}$ & A & C & T & $\mathrm{G}$ & $T$ \\
\hline MN12753 & VII & 2th passage culture & iv & $\mathrm{A}$ & C & 1 & $\mathrm{C}$ & $\bar{G}$ & $\mathrm{G}$ & $\mathrm{G}$ & A & & \begin{tabular}{|l|l}
$\mathrm{C}$ \\
\end{tabular} & $\mathrm{G}$ & 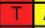 & $\mathrm{A}$ & \begin{tabular}{|l|}
$\mathrm{A}$ \\
\end{tabular} & \begin{tabular}{|l|l}
$\mathrm{C}$ \\
\end{tabular} & c & A & $\mathrm{C}$ & $\mathrm{A}$ & C & $\bar{T}$ & $\mathrm{G}$ & $T$ \\
\hline MN24049 & VIII & 4th passage culture & & A & C & T & C & G & G & $G$ & A & $T$ & $\begin{array}{ll}\text { C } \\
\end{array}$ & $G$ & & $\mathrm{~A}$ & A & $\begin{array}{ll}\text { C } \\
\end{array}$ & c & A & $\mathrm{N}$ & A & C & T & $\mathrm{G}$ & $T$ \\
\hline MN26006 & $\mathrm{IX}$ & 9th passage culture & & $\mathrm{A}$ & C & $\begin{array}{c}\mathrm{T} \\
\end{array}$ & $\bar{C}$ & $\bar{G}$ & $\bar{G}$ & $\mathrm{G}$ & $A$ & & \begin{tabular}{|l|l}
$\mathrm{C}$ \\
\end{tabular} & $\mathrm{G}$ & & $\mathrm{A}$ & A & \begin{tabular}{|l|l}
$\mathrm{C}$ & \\
\end{tabular} & \begin{tabular}{c|c}
$\mathrm{C}$ \\
$\mathrm{y}$
\end{tabular} & $\mathrm{A}$ & $\mathrm{C}$ & $\mathrm{A}$ & C & $\bar{T}$ & $\bar{G}$ & $T$ \\
\hline MN26063 & $x$ & 1th passage culture & & - & C & $\bar{T}$ & - & $\bar{G}$ & $\mathrm{G}$ & $\mathrm{G}$ & $\mathrm{A}$ & $\mathrm{T}$ & \begin{tabular}{|l|l}
$\mathrm{C}$ \\
\end{tabular} & $\mathrm{G}$ & $\bar{T}$ & \begin{tabular}{|l}
- \\
\end{tabular} & A & \begin{tabular}{|l|l}
$\mathrm{C}$ \\
\end{tabular} & c & A & $\mathrm{N}$ & A & $c$ & $\bar{T}$ & $G$ & $T$ \\
\hline MN23042a & $\bar{x}$ & 1th passage culture & & A & $\mathrm{C}$ & $\begin{array}{c}\mathrm{T} \\
\end{array}$ & $\mathrm{C}$ & $\bar{G}$ & $\mathrm{G}$ & $\mathrm{G}$ & $\mathrm{A}$ & & $\begin{array}{ll}\mathrm{C} & \mathrm{n} \\
\mathrm{C}\end{array}$ & $\mathrm{G}$ & & $\mathrm{A}$ & A & \begin{tabular}{|l|l}
$\mathrm{C}$ & \\
\end{tabular} & C & A & $\mathrm{C}$ & $A$ & C & & $\mathrm{G}$ & $\mathrm{G}$ \\
\hline
\end{tabular}

B Drug resistance loci

\begin{tabular}{|c|c|c|c|c|c|c|c|c|c|c|c|c|c|c|c|c|c|c|c|c|c|c|c|c|c|c|c|c|c|c|c|c|c|c|c|}
\hline \multirow[b]{2}{*}{10} & \multirow[b]{2}{*}{ assage level } & \multirow[b]{2}{*}{ I Passage history } & \multicolumn{8}{|c|}{ pfCRT } & \multicolumn{6}{|c|}{ pfMDR } & \multicolumn{4}{|c|}{ pfDHPS } & \multicolumn{4}{|c|}{ pfDHFR } & \multicolumn{3}{|c|}{ pfCYTB } & \multicolumn{3}{|c|}{ pfATPase } & \multicolumn{5}{|c|}{ Kelch K13 } \\
\hline & & & N & $\stackrel{N}{\Sigma}$ & $\begin{array}{l}\stackrel{2}{z} \\
z\end{array}$ & $\begin{array}{l}0 \\
z \\
\end{array}$ & के & ্ָণি & $\begin{array}{l}\stackrel{N}{N} \\
\text { లె }\end{array}$ & $\begin{array}{l}\mathscr{L} \\
\stackrel{\underline{m}}{ }\end{array}$ & $\begin{array}{l}\infty \\
\stackrel{\infty}{z} \\
\end{array}$ & $\stackrel{\varpi}{\check{\zeta}}$ & $\underset{\substack{\infty \\
\complement}}{\Sigma}$ & 志 & $\frac{\text { Jै }}{\text { ¿ }}$ & $\frac{\mathscr{0}}{\stackrel{N}{0}}$ & $\begin{array}{l}\mathscr{ల} \\
\text { ஸे }\end{array}$ & 旁 & 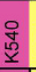 & $\begin{array}{l}\bar{\infty} \\
\stackrel{8}{8} \\
\stackrel{8}{\alpha}\end{array}$ & $\frac{5}{2}$ & ర్రి & $\underset{\Xi}{ \pm}$ & $\stackrel{\infty}{\circ}$ & $\begin{array}{l}\infty \\
\stackrel{્}{ர} \\
\end{array}$ & 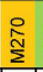 & ్ֻత్ & స్తి & 宂 & $\begin{array}{l}8 \\
0 \\
\omega \\
\text { ஸे }\end{array}$ & $\begin{array}{l}\text { రે } \\
0 \\
0 \\
0 \\
0\end{array}$ & 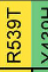 & $\begin{array}{l}\bar{T} \\
\stackrel{\rho}{y} \\
\succ\end{array}$ & $\begin{array}{l}\underline{\underline{m}} \\
\stackrel{\tilde{f}}{6} \\
\end{array}$ & \\
\hline TM90C2A & nd & WRAIR-Culture & $\mathrm{C}$ & 1 & $E$ & \begin{tabular}{|l|}
$\mathrm{T}$ \\
\end{tabular} & $\mathrm{H}$ & $s$ & $\mathrm{~s}$ & $\mathrm{~T}$ & $\mathrm{~N}$ & $\mathrm{Y}$ & $\mathrm{R}$ & \begin{tabular}{|l|}
$\mathrm{s}$ \\
\end{tabular} & \begin{tabular}{|l|}
$\mathrm{N}$ \\
\end{tabular} & $\mathrm{D}$ & $\mathrm{s}$ & $\mathrm{A}$ & $\mathrm{K}$ & A & \begin{tabular}{|l|l}
$Y$ & 1 \\
\end{tabular} & $\mathrm{M}$ & $L$ & $\mathrm{~A}$ & $E$ & $\mathrm{~S}$ & 1 & $\mathrm{R}$ & $\mathrm{L}$ & $\mathrm{N}(\mathrm{A})$ & C & \begin{tabular}{l|l}
$R$ & $Y$ \\
\end{tabular} & $Y$ & 1 & $\mathrm{D}$ \\
\hline M & & 17 & c & 1 & $E$ & $\mathrm{~T}$ & $\mathrm{H}$ & 5 & $\mathrm{~s}$ & $\mathrm{~T}$ & $\mathrm{~N}$ & $\mathrm{~F}$ & $\mathrm{R}$ & $\mathrm{s}$ & $\mathrm{N}$ & $\mathrm{D}$ & $s$ & $\mathrm{G}$ & $\mathrm{K}$ & \begin{tabular}{l|l} 
\\
\end{tabular} & 1 & $\mathrm{R}$ & $\mathrm{L}$ & iv & Y & $M$ & A & A & & $S$ & C & \begin{tabular}{l|l}
$\mathrm{R}$ & $\mathrm{V}$ \\
\end{tabular} & $Y$ & 1 & - \\
\hline $\mathrm{MN}$ & & & $\mathrm{C}$ & $\mathrm{I}$ & $E$ & $\mathrm{~T}$ & $\mathrm{H}$ & S & $\mathrm{s}$ & $\mathrm{T}$ & $\mathrm{N}$ & $\mathrm{F}$ & $\mathrm{R}$ & $\mathrm{s}$ & $\mathrm{N}$ & $\mathrm{D}$ & $\mathrm{s}$ & $\mathrm{G}$ & $\mathrm{K}$ & $G$ & 1 & $\mathrm{R}$ & $\mathrm{L}$ & $\mathrm{N}$ & $Y$ & $M$ & A & A & 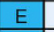 & S & C & $\mathrm{R}$ & $Y$ & 1 & - \\
\hline $\mathrm{MN}$ & & & c & 1 & $\mathrm{E}$ & $\mathrm{T}$ & $\mathrm{H}$ & $s$ & $\mathrm{~s}$ & $\mathrm{~T}$ & $\mathrm{~N}$ & $\mathrm{Y}$ & $\mathrm{R}$ & $\mathrm{s}$ & $\mathrm{N}$ & $\mathrm{D}$ & $\mathrm{s}$ & A & $\mathrm{K}$ & A & 1 & $\mathrm{R}$ & \begin{tabular}{l|l}
$L$ & $N$ \\
\end{tabular} & $N(A)$ & $Y$ & M & $\mathrm{L}$ & A & 드 & $\mathrm{S}$ & \begin{tabular}{|l|l} 
nd \\
\end{tabular} & \begin{tabular}{l|l}
$n d$ & $n$
\end{tabular} & \begin{tabular}{l|l}
$n d$ & $n$ \\
\end{tabular} & nd & n \\
\hline $\mathrm{MN}$ & 11 & Iture & C & 1 & $\mathrm{E}$ & \begin{tabular}{|l|}
$\mathrm{T}$ \\
\end{tabular} & $\mathrm{H}$ & $s$ & $\mathrm{~s}$ & $\mathrm{~T}$ & $\mathrm{~N}$ & $\mathrm{Y}$ & $\mathrm{R}$ & $\mathrm{s}$ & \begin{tabular}{|l|}
$\mathrm{N}$ \\
\end{tabular} & $\mathrm{D}$ & $\mathrm{s}$ & $\mathrm{A}$ & $\mathrm{K}$ & $\mathrm{A}$ & 1 & $\mathrm{R}$ & \begin{tabular}{|l|l|}
$\mathrm{L}$ & $\mathrm{N}$ \\
\end{tabular} & $N(A)$ & $\mathrm{Y}$ & $M$ & $\mathrm{~L}$ & A & $\mathrm{E}$ & S & nd & \begin{tabular}{l|l} 
nd & $r$ \\
\end{tabular} & \begin{tabular}{l|l}
$\mathrm{nd}$ & $\mathrm{n}$ \\
\end{tabular} & nd & nd \\
\hline $\mathrm{MN}$ & 1 & & $\mathrm{C}$ & 1 & $E$ & $\mathrm{~T}$ & $\mathrm{H}$ & $s$ & $\mathrm{~s}$ & $\mathrm{~T}$ & $\mathrm{~N}$ & $\mathrm{Y}$ & $\mathrm{R}$ & 5 & $\mathrm{~N}$ & $\mathrm{D}$ & $\mathrm{s}$ & A & $\mathrm{K}$ & $\mathrm{A}$ & 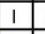 & $\mathrm{F}$ & \begin{tabular}{l|l}
$\mathrm{L}$ & $\mathrm{N}$ \\
\end{tabular} & $N(A)$ & $Y$ & $\mathrm{M}$ & L & A & 드 & S & nd & \begin{tabular}{l|l}
$n \mathrm{nd}$ & $\mathrm{r}$ \\
\end{tabular} & \begin{tabular}{l|l}
$\mathrm{nd}$ & $\mathrm{n}$ \\
\end{tabular} & nd & $\mathrm{n}$ \\
\hline & $v$ & & $\mathrm{C}$ & $\mathrm{I}$ & $E$ & $\mathrm{~T}$ & $\mathrm{H}$ & $S$ & $\mathrm{~s}$ & $\mathrm{~T}$ & $\mathrm{~N}$ & $\mathrm{Y}$ & $\mathrm{R}$ & $\mathrm{s}$ & $\mathrm{N}$ & $\mathrm{D}$ & $s$ & $\mathrm{~A}$ & $\mathrm{~K}$ & $\mathrm{~A}$ & 1 & $\mathrm{R}$ & \begin{tabular}{l|l}
$\mathrm{L}$ & $\mathrm{N}$ \\
\end{tabular} & $N(A)$ & $Y$ & $\mathrm{M}$ & $\mathrm{L}$ & \begin{tabular}{|l|l|}
$A$ & $N$ \\
\end{tabular} & $\mathrm{Neg}$. & $\underline{s}$ & nd & \begin{tabular}{l|l} 
& $\mathrm{n} d$ \\
\end{tabular} & \begin{tabular}{l|l}
$\mathrm{nd}$ & $\mathrm{n}$ \\
\end{tabular} & nd & \\
\hline & & & $\mathrm{C}$ & 1 & $\mathrm{E}$ & $\mathrm{T}$ & $\mathrm{H}$ & $s$ & $\mathrm{~s}$ & $\mathrm{~T}$ & $\mathrm{~N}$ & \begin{tabular}{|l|} 
\\
\end{tabular} & $\mathrm{R}$ & $\mathrm{s}$ & $\mathrm{N}$ & $\mathrm{D}$ & $\mathrm{s}$ & A & $\mathrm{K}$ & A & 1 & $\mathrm{R}$ & $\mathrm{L} / \mathrm{N}$ & $N(A)$ & $Y$ & $\mathrm{M}$ & $\mathrm{L}$ & A & $E$ & $S$ & nd & \begin{tabular}{l|l}
$n d$ & $r$
\end{tabular} & \begin{tabular}{l|l}
$n d$ & $n$ \\
\end{tabular} & nd & n \\
\hline MN2404 & & age culture & $c$ & 1 & $\mathrm{E}$ & $\mathrm{T}$ & $\mathrm{H}$ & $s$ & $\mathrm{~s}$ & $\mathrm{~T}$ & $\mathrm{~N}$ & \begin{tabular}{|l|}
$\mathrm{Y}$ \\
\end{tabular} & $\mathrm{R}$ & $S$ & $\mathrm{~N}$ & $\mathrm{D}$ & $\mathrm{s}$ & $\mathrm{A}$ & $\mathrm{K}$ & $\mathrm{A}$ & \begin{tabular}{|l|l|}
1 & \\
\end{tabular} & $\mathrm{R}$ & \begin{tabular}{l|l}
$\mathrm{L}$ & $\mathrm{N}$ \\
\end{tabular} & $N(A)$ & $Y$ & $\mathrm{M}$ & $\mathrm{L}$ & A & $E$ & $S$ & nd & \begin{tabular}{|l|l} 
nd & r \\
\end{tabular} & \begin{tabular}{l|l}
$n d$ & $n$ \\
\end{tabular} & nd & n \\
\hline & & ture & $\mathrm{C}$ & 1 & $E$ & $\mathrm{~T}$ & $\mathrm{H}$ & $\mathrm{s}$ & $\mathrm{s}$ & $\mathrm{T}$ & $\mathrm{N}$ & $\mathrm{Y}$ & $\mathrm{R}$ & $\mathrm{s}$ & $N$ & $\mathrm{D}$ & $\mathrm{s}$ & $\mathrm{A}$ & $\mathrm{K}$ & $\mathrm{A}$ & 1 & $\mathrm{R}$ & \begin{tabular}{l|l}
$\mathrm{L}$ & $\mathrm{N}$ \\
\end{tabular} & $N(A)$ & $\mathrm{Y}$ & $\mathrm{M}$ & $\mathrm{L}$ & A & 드 & $\mathrm{s}$ & nd & nd & \begin{tabular}{l|l}
$\mathrm{nd}$ & $\mathrm{n}$ \\
$\mathrm{n}$
\end{tabular} & nd & nd \\
\hline & & & C & 1 & E & $\mathrm{T}$ & $\mathrm{H}$ & $s$ & $s$ & \begin{tabular}{|l|}
$T$ \\
\end{tabular} & $\mathrm{~N}$ & \begin{tabular}{|l|}
$\mathrm{Y}$ \\
\end{tabular} & $\mathrm{R}$ & $s$ & $\mathrm{~N}$ & $D$ & $\mathrm{~s}$ & A & $\mathrm{K}$ & A & 1 & $R$ & \begin{tabular}{l|l} 
& $N$ \\
\end{tabular} & $N(A)$ & $Y$ & $\mathrm{M}$ & $\mathrm{L}$ & A & & 5 & C & $\mathrm{R}$ & $Y$ & 1 & D \\
\hline N23042 & $x$ & th passage culture & $c$ & 1 & $\mathrm{E}$ & 1 & $\mathrm{H}$ & & 0 & $\mathrm{~T}$ & $\mathrm{~N}$ & 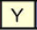 & $\mathrm{R}$ & 101 & $\mathrm{~N} \mid$ & 5 & 1 & $\mathrm{~A}$ & $\mathrm{~N}$ & A & 1 & $\mathrm{R}$ & \begin{tabular}{l|l}
$\mathrm{L}$ & $\mathrm{N}$ \\
\end{tabular} & $N(A)$ & $\mathrm{P}$ & $\mathrm{M}$ & 1 L & $A$ & L & 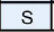 & \begin{tabular}{|l|} 
nd \\
\end{tabular} & \begin{tabular}{|l|l} 
nd & \\
\end{tabular} & \begin{tabular}{l|l}
$\mathrm{nd}$ & $\mathrm{n}$ \\
\end{tabular} & nd] & \\
\hline
\end{tabular}

Figure 3. Molecular barcode and antimalarial drug resistance loci across C2A passages. (A) Plasmodium falciparum barcode. (B) Drug resistance genotyping profiles. $n d=$ not determined; $\lambda=$ spleen intact.

a single genotype across passages, despite the observed $p f m d r 1 \mathrm{CNV}$, we performed genotyping using a molecular barcode assay that assesses the parasite genotype based on a combination of 24 neutral loci across the 14 P. falciparum chromosomes ${ }^{25}$. Genotyping by molecular barcode assay demonstrated that C2A parasites across all passages from TM90C2A to passage X show identical or very closely related major haplotypes (Fig. 3A). Genetic analysis of drug resistance loci revealed resistance mutations across all passages including a mutation in the in vitro-adapted TM90C2A within the $p f c r t$ (M74I, N75E, N76T, A220S, N326S, I356T) and $p f d h f r$ (N51I, C59R, I164L, S108N) loci (Fig. 3B). No mutations were found in the Kelch K13 locus across TM90C2A and passage II, III and $\mathrm{X}$. However, compared to the 3D7 reference sequence we found a tandem asparagine insert (NN) after codon 142 in the propeller domain in passages II, III and X as well as in the in vitro-adapted TM90C2A. This mutation has been observed in other parasites and is not related to an artemisinin resistance phenotype ${ }^{26}$. Interestingly specific mutations in $p f m d r 1$ (Y184F), pfdhps (A437G, A581G) and pfATPase (L263A) were only found in passages II and III (Fig. 3B). Similarly distinct sets of mutations were found in the in vitro-adapted TM90C2A in pfdhfr (N51Y, C59M) and pfATPase (L263I, A623R, I431L, S769A/N), and these parasites also harbor unique mutations in $p f c y t b$ (Y268E, M270S). Given the molecular barcode data these observations suggest that the original C2A strain was a mixture of closely related parasite genotypes from which at least two were selected during subsequent Aotus passages (represented by passages II/III and passages IV-X, respectively) and another genotype was selected during in vitro adaptation (TM90C2A).

Serial passaging of C2A in Aotus selects for increased growth phenotype. As in other biological systems $P$. falciparum adaptation (in vitro and in animals) selects for the genotypes with maximal growth in the absence of any other selection (i.e., drug pressure ${ }^{27-29}$. Increased growth rates typically result in decreased drug susceptibility profiles as demonstrated in the rodent malaria mode ${ }^{30}$. We therefore wanted to quantify the altered parasite growth rates across Aotus passages, as a possible contributor to the observed changes in drug susceptibility. We analyzed growth rates during Aotus adaptation based on the recorded parasitemia from each of the 10 passage experiments (Fig. 4A), considering the time (Fig. 4B) and peak parasitemia (Fig. 4C). Specifically we estimated parasite multiplication rate (PMR, Fig. 4D) from the median of all increases in parasitemia at 48-hour intervals averaged across monkeys in the same passage. These data demonstrated that parasite growth increased significantly from a PMR just above 1 in passage I to PMR of above 7 in passage X. Interestingly increased growth rates were not linked to genomic deletions on chromosomes 2 and 9 (Fig. S1) that occur frequently during in vitro parasite adaptation ${ }^{31}$ and have been observed phenotypically in a splenectomized monkey model ${ }^{32}$.

\section{Discussion}

In a previous study we observed MQ and AS treatment failures with the Thai multi-drug resistant PfC2A in Aotus monkeys ${ }^{15}$. Here we systematically investigate the antimalarial drug responses of this isolate and its changes during host adaptation.

Artemisinin resistance in humans is defined as reduced parasite clearance rate or persistence of microscopically detectable parasites on the third day of ACT therapy ${ }^{33}$. We observed suppression and persistence of 
A

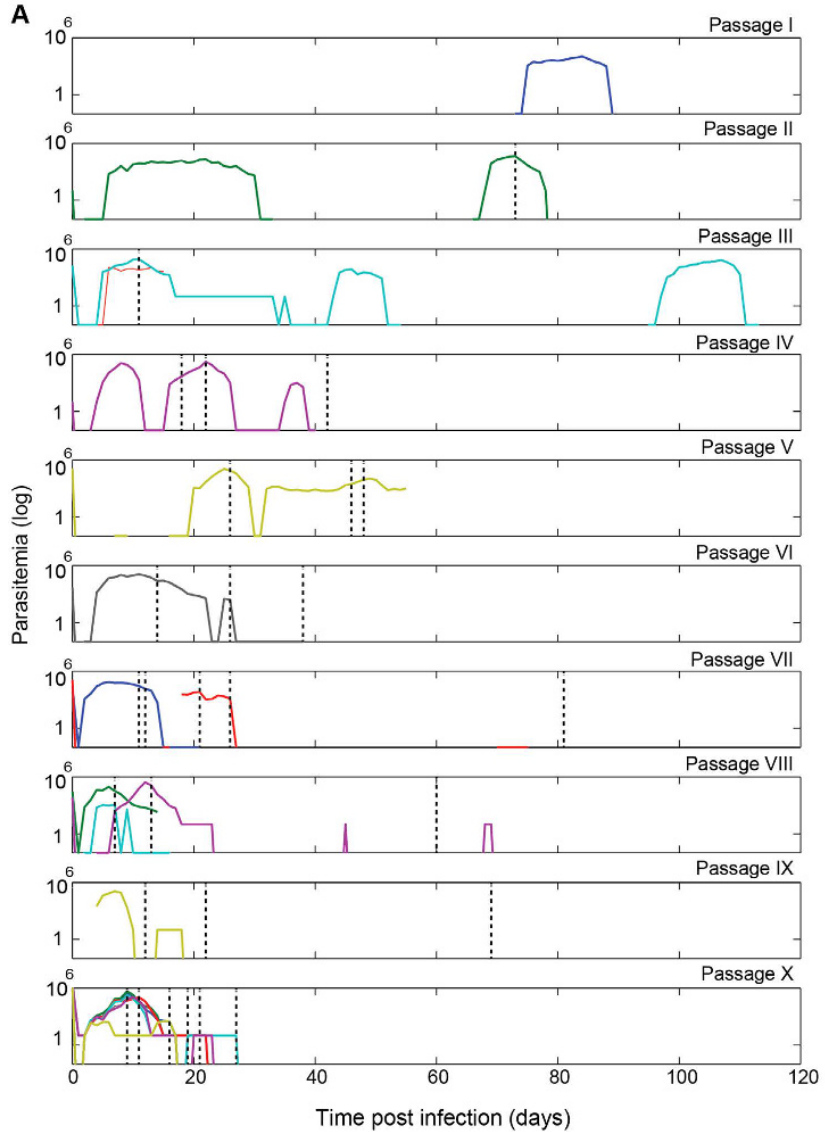

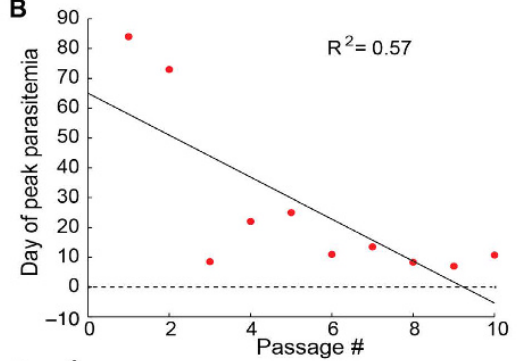
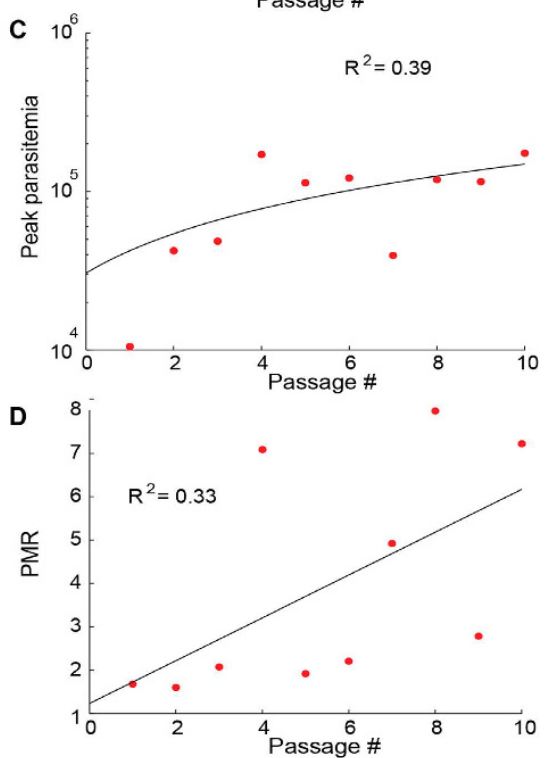

Figure 4. Plasmodium falciparum C2A growth across Aotus passages. (A) Parasitemia plots from individual monkeys (colored lines) with time of treatment (black dotted lines) across each passage. Parasitemia was followed for up to 100 days post inoculation and frequently fell below detectability (disappearance of colored lines). (B) Day of peak parasitemia trend. The day of peak parasitemia is the time since inoculation when peak parasitemia was achieved, averaged across monkeys for each passage. (C) Peak parasitemia trend. The value of peak parasitemia is the maximum parasitemia reached over the course of infection, averaged across monkeys for each passage. (D) Parasite multiplication rate (PMR). The PMR is the median of parasitemia increases across 48-hr periods over the course of an infection, averaged across monkeys for each passage. The data is modeled with linear regression.

microscopically detectable parasites beyond the third day of oral AS alone or in combination with MQ, indicating resistance of the Aotus-adapted PfC2A isolate based on the above definition. The observed phenotype is unlikely due to limited AS and/or MQ bioavailability by the oral delivery route as our previous experiments in Aotus with the FVO P. falciparum strain have efficiently cleared parasites at day 4 when AS was administered i.v. or orally at $8 \mathrm{mg} / \mathrm{Kg}$ for three days ${ }^{34}$. Similarly MQ treatment as a single dose of $20 \mathrm{mg} / \mathrm{Kg}$ cleared parasites on day 5 following initiation of treatment in Aotus infected with $\mathrm{FVO}^{35}$. However, we cannot exclude that an inoculum effect ${ }^{36}$ may have contributed to the observed relative lower efficiency of the oral route at high parasitemia densities $\left(\geq 100 \times 10^{3} / \mu \mathrm{L}\right)$ compared to the i.v. route at low parasitemia $\left(<1.0 \times 10^{3} / \mu \mathrm{L}\right)$. Finally, splenectomy may have resulted in reduced efficiency of artemisinin-mediated parasite clearance as discussed previously ${ }^{35}$.

In vitro and ex vivo experiments demonstrated reduced DHA susceptibility at later Aotus passages, although remaining below the standard resistance threshold of $1 \%$ ring stage survival. Genotyping experiments demonstrated presence of wild type Kelch K13 locus across all C2A passages, therefore ruling out involvement of this major resistance locus in the observed in vivo and in vitro or ex vivo drug susceptibility phenotypes. We also demonstrated treatment failure upon MQ treatment in Aotus and MQ drug resistant phenotype in vitro and ex vivo. Pfmdr 1 copy number fluctuated between 2 to 2.5 during Aotus adaptation while MQ susceptibility decreased at later passages. These observations contrast with in vitro studies where $p f m d r 1$ de-amplification results in increased MQ susceptibility. For example, in an artelinic acid (AL)-resistant line of $P$. falciparum (W2AL80) and clones originating from it, $p f m d r 1$ de-amplification resulted in partial reversal of resistance to $\mathrm{AL}$ and increased susceptibility to MQ, even in the absence of drug pressure ${ }^{37,38}$.

It is possible that both, reduced artemisinin susceptibility and MQ resistance during Aotus adaptation are due to increased growth rates. For example, treatment of neurosyphilis with the zoonotic malaria parasite P. knowlesi was eventually abandoned due to increased virulence of this species after multiple passages in humans ${ }^{39}$. We modeled PfC2A growth rates during Aotus adaptation and indeed demonstrated a significant increase in parasite 


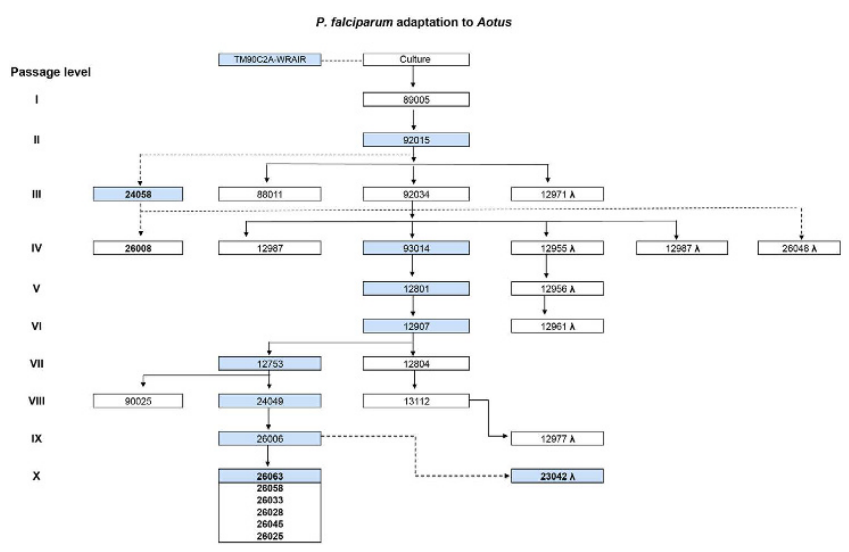

Figure 5. Genealogy of Plasmodium. falciparum C2A adaptation to Aotus lemurinus lemurinus monkeys. Blue highlighted squares indicate samples from which gDNA was extracted for genetic studies. Aotus monkey numbers in bold mark those that have been used in this study.

multiplication rate from near 1 (passage I) to above 7 (passage X). It is therefore possible that the increased growth (i.e., parasite fitness) during serial passaging in Aotus resulted in altered drug susceptibility phenotypes. Similar observations have been made in the mouse malaria model, where increased fitness in $P$. chabaudi correlated with reduced susceptibility to artemisinin ${ }^{30}$.

In conclusion, we present a comprehensive study on Aotus adaptation of the multidrug resistant $P$. falciparum C2A strain. Combined phenotypic and molecular analysis of parasites across Aotus passages demonstrates that host adaptation has co-selected for reduced drug susceptibility in this non-human primate model. Our data also suggest that this model may be used for the evaluation of anti-malarial drugs against the recently detected Artemisinin resistant strains emerging from South-East Asia.

\section{Materials and Methods}

Plasmodium falciparum parasite strains. The original $P$. falciparum TM90C2A strain and subsequent passages in Aotus (Fig. 5) ${ }^{15}$, as well as ex vivo cultures thereof, were used in this study. In addition, the Aotus adapted strain $P$. falciparum $\mathrm{FVO}^{34,40}$ and in vitro adapted strains, D6, D10, CS2 and W2mef, were used as control parasites for these studies.

Animals. Monkeys of the species Aotus l. lemurinus (Panamanian Owl monkey) Karyotype VIII \& IX ${ }^{41}$ were used in this study. Animals were housed at Gorgas Memorial Institute of Health Studies (ICGES) in Panama, and cared and maintained as described ${ }^{42}$. Briefly, the animals were kept in stainless steel 4 unit quads cages (Lab Products Inc., Seaford, DE) with dimensions of $27 \times 23.5 \times 29.5$ inches. Each cage was fitted with a 3/4-inch-diameter PVC pipe perch placed across $2 / 3$ of the length of the cage and a 6-inch-diameter $\times 14.5$ inches long PVC T pipe nest-box attached to the roof and back of the cage with cable zip ties. Cages were routinely cleaned and sterilized at $180^{\circ} \mathrm{F}$ at weekly intervals in a cage washing machine (Steris ${ }^{\circledR}$, Erie, PA). During experimental infections the animals did not receive analgesics.

The experimental protocol was approved by the ICGES Institutional Laboratory Animal Care and Use Committee (CIUCAL) in accordance with procedures described in the "Guide for the Care and Use of Laboratory Animals," 1996; protocol approval number 2006/02. All experiments described herein were performed in accordance with the approved guidelines.

Drug efficacy studies in Aotus monkeys. To determine drug efficacy using the commonly used delivery route in humans, MQ and AS were applied orally alone or in combination. Six splenectomized male and female laboratory bred Aotus weighing between $758-829 \mathrm{~g}$ and one spleen intact control were inoculated intravenously (i.v.) in the saphenous vein with $5 \times 10^{6}$ parasitized erythrocytes of the C2A strain from a donor monkey (passage IX). The animals were splenectomized $\sim 30$ days prior to inoculation as described ${ }^{43}$ (Fig. 5, Table 1). Parasite density was determined daily by Giemsa stained thick blood smears using the method described by Earle and Perez ${ }^{44}$, with $50 \mu \mathrm{L}$ of blood obtained with a lancet prick from the marginal ear vein. When parasitemia reached $\sim 100,000$ infected RBCs/ $\mu \mathrm{L}$, animals were assigned to one of the three treatment arms (groups) by weight and sex and treated as shown in Table S3. Drug doses were calculated on a milligram (mg) base per kilogram (kg) of body weight basis ${ }^{45}$. Oral administration of drugs was by gastric intubation with a 14 French red rubber catheter, while for i.v. administration a $25 \mathrm{~g}$ butterfly needle was used. Response to treatment was categorized as no effect, parasite suppression without clearance, parasite clearance and recrudescence, or parasite clearance and cure. The day of parasite clearance was defined as the first of three consecutive days in which the thick blood films were parasite negative. The day of recrudescence was defined as the first of 3 consecutive days of positive thick blood films after a period of clearance. Parasite suppression was defined as a transient decrease in parasite density post-treatment without clearance ${ }^{15}$. Animals were considered cured if no recrudescence was observed after parasite clearance and 100 days of follow up with twice a week negative thick smears. 
Before initiation of treatment, citrated whole blood was collected from each animal, plasma removed, and cryopreserved with Glycerolyte ${ }^{\circledR}$ in liquid nitrogen and labeled passage level X for further in vitro $\mathrm{IC}_{50} \mathrm{~s}$ determination, Ring Susceptibility Assay (RSA) and genotypic studies. Rescue treatment with MQ at 40mg/Kg orally once and AS at $20 \mathrm{mg} / \mathrm{Kg}$ i.v. for three days was triggered by any of the following: Hematocrit (HTO) below $50 \%$ of baseline, thrombocytopenia $\left(<50 \times 10^{3} / \mu \mathrm{L}\right)$ or signs of depression or anorexia as determined by the attending veterinarian. Two animals were also included in this study as historical references representing infections with C2A passages III (MN24058) and IV (MN26008) shown in Fig. 5 and Table S1.

In vitro drug assays. In vitro $\mathrm{IC}_{50}$ values were obtained using the hypoxanthine incorporation assay ${ }^{46}$. $\mathrm{IC}_{50}$ cutoff values indicative of resistance were adopted from the literature as follows: $>100 \mathrm{nM}$ for $\mathrm{CQ}^{21-23,47}, 30 \mathrm{nM}$ for $\mathrm{MQ}^{21,22,47}, 20 \mathrm{nM}$ for $\mathrm{AS}^{21,23}$, and $12 \mathrm{nM}$ for $\mathrm{DHA}^{21}$. Resistance cutoff points for QHS and ATV were not available from the literature. Ex vivo parasite passages were cultured for up to 8 serial passages in RPMI media containing $25 \%$ human $\mathrm{AB}+$ serum and $4 \%$ packed red blood cells. Thin smears were stained with Giemsa for morphological studies and detection of gametocytes. In vitro ring stage $0-3$ hours survival assays (RSA) were carried out as described ${ }^{24}$ and the threshold for resistance was established at $1 \%$ survival.

Genetic analysis of parasite strains. Molecular barcode and drug resistance genotyping. Genomic DNA was obtained from blood samples using phenol extraction-ethanol precipitation protocol for purification and concentration of DNA as described ${ }^{48}$. DNA concentrations of the samples were measured using a NanoDrop ${ }^{\circledR}$ spectrophotometer ND1000. Extracted DNA was pre-amplified and genotyped across 24 genomic loci with both high-resolution melting (HRM) and TaqMan technologies (Life Technologies, Grand Island, NY) as previously described $^{25,49}$. Samples were diluted 1:20 and $5 \mu \mathrm{L}$ of each pre-amplified product used for the molecular barcode. TaqMan barcoding assays were run on a ViiA system (Applied Biosystems) and genotypes called using the pre-installed analysis software. To confirm haplotypes and determine drug resistance genotypes of the C2A Aotus passages, we used a high resolution melting (HRM) analysis as described ${ }^{50}$.

Pfmdr1 copy number variation (CNV). To determine CNV in the $p f m d r 1$ gene across C2A Aotus-adapted passages, gDNA was extracted and used in a qPCR assay, as described ${ }^{13}$. Pfmdr1 fold changes between C2A passage levels III-X and reference strain FVO were calculated as described ${ }^{51}$. Fold change results were rounded to the next significant integer for the purpose of determining copy number. Primer sequences are included in Table S2.

Gene sequencing. To determine single nucleotide polymorphisms (SNPs) in the K13 propeller domain, PCR sequencing was carried out. The full ORF of K13 was PCR amplified using Phusion HF DNA Polymerase kit and primers $1 \mathrm{~F}$ and $1 \mathrm{R}$. The resulting PCR product of $\sim 2.2 \mathrm{~kb}$ was purified by gel extraction (QIAquick Gel Extraction Kit, Qiagen) and sequenced at Genewiz using primers 1F, 2F, 3F, 1R, 2R and 3R. Primer sequences are included in Table S2.

Chromosomal deletions. To determine whether common genomic deletions in the subtelomeric regions of either chromosome 2 or 9 occurred during C2A passages in Aotus, several loci were analyzed by PCR. For this purpose genomic DNA was extracted using Qiagen ${ }^{\mathrm{TM}}$ DNA kit across $P$. falciparum C2A Aotus-adapted passages, as well as $P$. falciparum reference strains FVO, D10 and 3D7. PCR amplification of genes PF3D7_0201500 (PFB0075c) and PF3D7_0202000 (PFB0100c) on chromosome 2, as well as PF3D7_0935400 (PFI1710w), PF3D7_0936300 (PFI1755c), PF3D7_0936800 (PFI1780w) on chromosome 9 were carried out in a BioRad ${ }^{\mathrm{TM}}$ PCR machine using the following amplification program: $95^{\circ}$ Celsius $(\mathrm{C}) \times 5$ minutes, $95^{\circ} \mathrm{C} \times 30$ minutes, $51.4^{\circ} \mathrm{C} \times 30$ minutes, $61.0^{\circ} \mathrm{C} \times 3$ minutes for 35 cycles, plus $72^{\circ} \mathrm{C} \times 10$ minutes. Amplicons were loaded onto a $1 \%$ agarose gel and subjected to electrophoresis at 150 Volts for 30 minutes, stained with ethidium bromide and photo-documentation done with a UV light reader. Primer sequences are included in Table S2; primers for KAHRP (PFB0100c) amplification were published previously ${ }^{52}$.

Statistical analysis. Prism 5 Graph Pad ${ }^{\circledR}$ Software was used to plot and calculate the mean and standard error of the mean (SEM), and the JMP ${ }^{\circledR}$ Pro 10.0.0 statistical program (SAS Institute Inc, Middleton, MA) was used for the Mann-Whitney U significance test between unpaired $\mathrm{IC}_{50}$ values for TM90C2A and passage levels II and X. Matlab R2013a was used to plot parasitemia curves and calculate parasite multiplication rate (PMR). PMR is modeled via polynomial regression using the polynomial degree corresponding to the lowest Akaike Information Criteria.

\section{References}

1. Alonso, P. L. et al. A research agenda to underpin malaria eradication. PLoS medicine 8, e1000406, doi: 10.1371/journal. pmed.1000406 (2011).

2. Sibley, C. H. Understanding artemisinin resistance. Science 347, 373-374 (2015).

3. Price, R. N. et al. Mefloquine resistance in Plasmodium falciparum and increased pfmdr1 gene copy number. Lancet 364, 438-447, doi: 10.1016/S0140-6736(04)16767-6 (2004).

4. Dondorp, A. M. et al. Artemisinin resistance in Plasmodium falciparum malaria. N Engl J Med 361, 455-467, doi: 10.1056/ NEJMoa0808859 (2009).

5. Takala-Harrison, S. et al. Independent Emergence of Artemisinin Resistance Mutations Among Plasmodium falciparum in Southeast Asia. J Infect Dis, doi: 10.1093/infdis/jiu491 (2014).

6. Ariey, F. et al. A molecular marker of artemisinin-resistant Plasmodium falciparum malaria. Nature 505, 50-55, doi: 10.1038/ nature12876 (2014)

7. Miotto, O. et al. Genetic architecture of artemisinin-resistant Plasmodium falciparum. Nat Genet, doi: 10.1038/ng.3189 (2015).

8. Amaratunga, C. et al. Plasmodium falciparum founder populations in western Cambodia have reduced artemisinin sensitivity in vitro. Antimicrob Agents Chemother 58, 4935-4937, doi: 10.1128/AAC.03055-14 (2014). 
9. Morris, C. A. et al. Effects of body size and gender on the population pharmacokinetics of artesunate and its active metabolite dihydroartemisinin in pediatric malaria patients. Antimicrob Agents Chemother 57, 5889-5900, doi: 10.1128/AAC.00635-13 (2013).

10. Edstein, M. D. et al. Antimalarial pharmacodynamics and pharmacokinetics of a third-generation antifolate-JPC2056-in cynomolgus monkeys using an in vivo in vitro model. J Antimicrob Chemother 60, 811-818, doi: 10.1093/jac/dkm280 (2007).

11. Looareesuwan, S. et al. Clinical studies of atovaquone, alone or in combination with other antimalarial drugs, for treatment of acute uncomplicated malaria in Thailand. Am J Trop Med Hyg 54, 62-66 (1996).

12. Woodrow, C. J. \& Bustamante, L. Y. Mechanisms of artemisinin action and resistance: wider focus is needed. Trends Parasitol 27, 2-3; author reply 3-4, doi: 10.1016/j.pt.2010.10.002 (2011).

13. Chavchich, M. et al. Role of pfmdr1 amplification and expression in induction of resistance to artemisinin derivatives in Plasmodium falciparum. Antimicrob Agents Chemother 54, 2455-2464, doi: 10.1128/AAC.00947-09 (2010).

14. Young, M. D., Porter, J. A., Jr. \& Johnson, C. M. Plasmodium vivax transmitted from man to monkey to man. Science 153, 1006-1007 (1966).

15. Obaldia, N., 3rd, Milhous, W. \& Kyle, D. Adaptation of a Thai multidrug-resistant C2A clone of Plasmodium falciparum to Aotus monkeys and its preliminary in vivo antimalarial drug efficacy-resistance profile. Am J Trop Med Hyg 81, 587-594, doi: 10.4269/ ajtmh.2009.08-0445 (2009).

16. Stepniewska, K. et al. In vivo parasitological measures of artemisinin susceptibility. J Infect Dis 201, 570-579, doi: 10.1086/650301 (2010).

17. Wilairatana, P. et al. An open randomized clinical trial of Artecom vs artesunate-mefloquine in the treatment of acute uncomplicated falciparum malaria in Thailand. Southeast Asian J Trop Med Public Health 33, 519-524 (2002).

18. Vijaykadga, S. et al. Delayed Plasmodium falciparum clearance following artesunate-mefloquine combination therapy in Thailand, 1997-2007. Malar J 11, 296, doi: 10.1186/1475-2875-11-296 (2012).

19. Eddleston, M., Davidson, R., Brent, A. \& Wilkinson, R. Oxford Handbook of Tropical Medicine. 56-61 (Oxford Universtiy Press Inc., 2008).

20. Reagan-Shaw, S., Nihal, M. \& Ahmad, N. Dose translation from animal to human studies revisited. Faseb J 22, 659-661, doi: 10.1096/f.07-9574LSF (2008).

21. Quashie, N. B. et al. A SYBR Green 1-based in vitro test of susceptibility of Ghanaian Plasmodium falciparum clinical isolates to a panel of anti-malarial drugs. Malar J 12, 450, doi: 10.1186/1475-2875-12-450 (2013).

22. Basco, L. Field application of in vitro assays for the sensitivity of human malaria parasites to antimalarial drugs. (WHO, 2007).

23. Toure, A. O. et al. [In vitro susceptibility of P. falciparum isolates from Abidjan (Cote d'Ivoire) to quinine, artesunate and chloroquine]. Sante 18, 43-47, doi: 10.1684/san.2008.0103 (2008).

24. Witkowski, B. et al. Novel phenotypic assays for the detection of artemisinin-resistant Plasmodium falciparum malaria in Cambodia: in-vitro and ex-vivo drug-response studies. Lancet Infect Dis 13, 1043-1049, doi: 10.1016/S1473-3099(13)70252-4 (2013).

25. Daniels, R. et al. A general SNP-based molecular barcode for Plasmodium falciparum identification and tracking. Malar J 7, 223, doi: 10.1186/1475-2875-7-223 (2008).

26. Torrentino-Madamet, M. et al. Limited polymorphisms in k13 gene in Plasmodium falciparum isolates from Dakar, Senegal in 20122013. Malar J 13, 472, doi: 10.1186/1475-2875-13-472 (2014).

27. Walliker, D., Hunt, P. \& Babiker, H. Fitness of drug-resistant malaria parasites. Acta Trop 94, 251-259, doi: 10.1016/j. actatropica.2005.04.005 (2005).

28. Gimode, W. R. et al. Fitness cost of resistance for lumefantrine and piperaquine-resistant Plasmodium berghei in a mouse model. Malar J 14, 38, doi: 10.1186/s12936-015-0550-5 (2015).

29. Eber, D. In Evolutionary Biology of Host-Parasite Relationship: Theory Meets Reality (eds R. Poulin, S. Morand \& A. Skorping) 163-184 (Elsevier Science B.V., 2000).

30. Schneider, P. et al. Virulence, drug sensitivity and transmission success in the rodent malaria, Plasmodium chabaudi. Proc Biol Sci 279, 4677-4685, doi: 10.1098/rspb.2012.1792 (2012).

31. Carret, C. K. et al. Microarray-based comparative genomic analyses of the human malaria parasite Plasmodium falciparum using Affymetrix arrays. Mol Biochem Parasitol 144, 177-186 (2005).

32. David, P. H., Hommel, M., Miller, L. H., Udeinya, I. J. \& Oligino, L. D. Parasite sequestration in Plasmodium falciparum malaria: spleen and antibody modulation of cytoadherence of infected erythrocytes. Proceedings of the National Academy of Sciences of the United States of America 80, 5075-5079 (1983).

33. Krishna, S. \& Kremsner, P. G. Antidogmatic approaches to artemisinin resistance: reappraisal as treatment failure with artemisinin combination therapy. Trends Parasitol 29, 313-317, doi: 10.1016/j.pt.2013.04.001 (2013).

34. Ohrt, C. et al. Efficacy of intravenous methylene blue, intravenous artesunate, and their combination in preclinical models of malaria. Malar J 13, 415, doi: 10.1186/1475-2875-13-415 (2014).

35. Buffet, P. A. et al. Ex vivo perfusion of human spleens maintains clearing and processing functions. Blood 107, 3745-3752, doi: 10.1182/blood-2005-10-4094 (2006).

36. Duraisingh, M. T. et al. Inoculum effect leads to overestimation of in vitro resistance for artemisinin derivatives and standard antimalarials: a Gambian field study. Parasitology 119 (Pt 5), 435-440 (1999).

37. Chen, N. et al. Deamplification of pfmdr1-containing amplicon on chromosome 5 in Plasmodium falciparum is associated with reduced resistance to artelinic acid in vitro. Antimicrob Agents Chemother 54, 3395-3401, doi: 10.1128/AAC.01421-09 (2010).

38. Tucker, M. S., Mutka, T., Sparks, K., Patel, J. \& Kyle, D. E. Phenotypic and genotypic analysis of in vitro-selected artemisinin-resistant progeny of Plasmodium falciparum. Antimicrob Agents Chemother 56, 302-314, doi: 10.1128/AAC.05540-11 (2012).

39. Coatney, G. R., Collins, W. E., Warren, M. \& Contacos, P. G. The Primate Malarias. (US Government Print Off, 1971).

40. Obaldia, N., 3rd et al. Evaluation of artemisone combinations in Aotus monkeys infected with Plasmodium falciparum. Antimicrob Agents Chemother 53, 3592-3594, doi: 10.1128/AAC.00471-09 (2009).

41. Ma, N. S. et al. Banding patterns of the chromosomes of two new karyotypes of the owl monkey, Aotus, captured in Panama. J Med Primatol 7, 146-155 (1978).

42. Obaldia, N., 3rd, Otero, W., Marin, C., Aparicio, J. \& Cisneros, G. Long-term effect of a simple nest-box on the reproductive efficiency and other life traits of an Aotus lemurinus lemurinus monkey colony: an animal model for malaria research. J Med Primatol 40, 383-391, doi: 10.1111/j.1600-0684.2011.00489.x (2011).

43. Gramzinski, R. A. et al. Susceptibility of Panamanian Aotus lemurinus lemurinus to sporozoite-induced Plasmodium falciparum (Santa Lucia) infection. Am J Trop Med Hyg 61, 19-25 (1999).

44. Earle, W. \& Perez, M. Enumeration of parasites in the blood of malaria patients. Journal of Laboratory and Clinical Medicine 17, $1124-1130$ (1932).

45. Rossan, R. N. In Antimalarials Drugs I: Biological background, experimental methods, and drug resistane (eds W. Peters \& WHG. Richards) Ch. 9, 265-280 (Springer-Verlag, 1984).

46. Desjardins, R. E., Canfield, C. J., Haynes, J. D. \& Chulay, J. D. Quantitative assessment of antimalarial activity in vitro by a semiautomated microdilution technique. Antimicrob Agents Chemother 16, 710-718 (1979).

47. Ndong, J. M. et al. In vitro activity of chloroquine, quinine, mefloquine and halofantrine against Gabonese isolates of Plasmodium falciparum. Trop Med Int Health 8, 25-29 (2003).

48. Moore, D. \& Dowhan, D. Purification and concentration of DNA from aqueous solutions. Curr Protoc Mol Biol Chapter 2, Unit 2 1A, doi: 10.1002/0471142727.mb0201as59 (2002). 
49. Mharakurwa, S. et al. Pre-amplification methods for tracking low-grade Plasmodium falciparum populations during scaled-up interventions in Southern Zambia. Malar J 13, 89, doi: 10.1186/1475-2875-13-89 (2014).

50. Daniels, R. et al. Rapid, field-deployable method for genotyping and discovery of single-nucleotide polymorphisms associated with drug resistance in Plasmodium falciparum. Antimicrob Agents Chemother 56, 2976-2986, doi: 10.1128/AAC.05737-11 (2012).

51. Livak, K. J. \& Schmittgen, T. D. Analysis of relative gene expression data using real-time quantitative PCR and the 2(-Delta Delta C(T)) Method. Methods 25, 402-408, doi: 10.1006/meth.2001.1262 (2001).

52. Marti, M., Good, R. T., Rug, M., Knuepfer, E. \& Cowman, A. F. Targeting malaria virulence and remodeling proteins to the host erythrocyte. Science 306, 1930-1933 (2004).

\section{Acknowledgements}

The authors wish to thank the Directors of the Gorgas Memorial Institute in Panama City, Drs. Jorge Motta and Nestor Sosa; Gines Sanchez and Gladys Calvino at Tropical Medicine Research in Panama City for administrative support; Maritza Brewer for secretarial assistance; Camilo Marin, Temistocles Gonzales and the animal care takers for their assistance with animal handling and care. We thank Katelyn Durfee for help with the molecular barcoding and drug resistance marker typing and Courtney Edison for help with the kelch13 propeller domain sequencing. This work was supported in part by a USAMDA contract W81XWH-07-C-044, a SENACYTIFHARU Panama doctoral fellowship to Nicanor Obaldia III, the Sistema Nacional de Investigacion of Panama (SNI) and the Department of Immunology and Infectious Diseases, Harvard T.H. Chan School of Public Health, Boston, MA, USA. LC and COB were supported by award number U54GM088558 from the National Institute of General Medical Sciences. The content is solely the responsibility of the authors and does not necessarily represent the official views of the National Institute of General Medical Sciences or the National Institutes of Health.

\section{Author Contributions}

N.O., G.S.D., W.O. and D.K. planned and executed Aotus experiments; N.O., M.M., W.O. and G.O.D. collected and analyzed Aotus monkey experimental data. N.O., L.G., S.K.V., A.M. and M.M. planned, executed and analyzed in vitro drug susceptibility assays. N.O., S.K.V., M.M., R.D., N.B., A.M. and P.Y.M. planned, executed and analyzed molecular biology and genotyping studies. N.O., L.M.C., C.B. and M.M. planned, analyzed and developed growth phenotype models. N.O., M.M., S.K.V., M.T.D. and D.F.W. designed the study. N.O. and M.M. wrote the paper with input from all coauthors.

\section{Additional Information}

Supplementary information accompanies this paper at http://www.nature.com/srep

Competing financial interests: The authors declare no competing financial interests.

How to cite this article: Obaldía, N. et al. Altered drug susceptibility during host adaptation of a Plasmodium falciparum strain in a non-human primate model. Sci. Rep. 6, 21216; doi: 10.1038/srep21216 (2016).

(i) This work is licensed under a Creative Commons Attribution 4.0 International License. The images or other third party material in this article are included in the article's Creative Commons license, unless indicated otherwise in the credit line; if the material is not included under the Creative Commons license, users will need to obtain permission from the license holder to reproduce the material. To view a copy of this license, visit http://creativecommons.org/licenses/by/4.0/ 